\title{
Low Salt Diet Is Associated with the Prevention of Exacerbation in Patients with Relapsing Remitting MS
}

\author{
Vera Kolyovska ${ }^{1}$, Velichka Pavlova ${ }^{1}$, Ivan Iliev ${ }^{1}$, Sava Todorov ${ }^{1}$ and Dimitar Maslarov ${ }^{2}$ \\ 1. Department of Experimental Morphology, Institute of Experimental Morphology, Pathology and Anthropology with Museum, \\ Bulgarian Academy of Sciences, Sofia 1113, Bulgaria \\ 2. Neurology Clinics, First MHAT, Sofia 1000, Bulgaria
}

\begin{abstract}
A marked increase in the incidence of autoimmune diseases in the past half-a-century has been observed. MS (multiple sclerosis) is one of the most common chronic immune-mediated inflammatory diseases of the central nervous system in young adults which is characterized by inflammatory demyelination and neurodegeneration with unpredictable effects lasting for the rest of their lives. The aim of the MS treatment is to prevent the demyelination and the reduction of axonal loss. Drugs approved for use in MS which reduce the frequency of exacerbations or slow disability progression are referred as DMDs (disease-modifying drugs). Additional treatments of MS include: dietary supplementation, herbal medicine intake, vitamin D3 and vitamin B supplementation and relaxation techniques. However, little is known about the environmental factors that directly influence Th17 cells. Increased salt (sodium chloride) concentrations found locally under physiological conditions in vivo markedly boost the induction of murine and human Th17 cells. Pathogenic IL-23-dependent Th17 cells have been shown to be critical for the development of EAE (experimental autoimmune encephalomyelitis) along with some genetic risk factors.
\end{abstract}

Key words: Multiple sclerosis, exacerbation, salt, diet.

\section{Introduction}

Recently, at "MS Boston 2014", the 2014 Joint ACTRIMS/ECTRIMS (Americas and European Committees for Treatment and Research in Multiple Sclerosis) meeting, few publications about sodium intake and correlation with increased disease activity in MS (multiple sclerosis) [1] and in other autoimmune diseases were presented.

Humans with MS having higher sodium intake showed approximately three times higher risk for exacerbations in symptoms as well as disease activity, compared to those with lower sodium intake, a new study showed [2]. Additionally, hypercaloric Western-style diets, characterized by high salt and animal fat, red meat, sugar-sweetened drinks, fried food, low fiber, and lack of physical exercise increase inflammation [3]. Magnesium deficiency may

Corresponding author: Vera Kolyovska, Ph.D., assistant professor, research fields: immunology and biochemistry. E-mail: verakol@abv.bg. contribute to a wide range of pathologies: hypertension, peroxynitrite damage (migraine, multiple sclerosis, glaucoma, Alzheimer's disease, etc.), recurrent bacterial infection due to low levels of nitric oxide in the cavities or fungal infections due to a depressed immune system. It is also associated with $\mathrm{Ca}$ deficiency (osteoporosis, hypertension, mood swings), hearing loss, diabetes type II, cramps, muscle weakness or impotence [4, 5]. However, the Argentinean authors were cautious about drawing conclusions. That small observational study suggested a positive correlation between sodium intake and MS disease activity [6]. Thus, a new possibility to address the effect of sodium intake in MS patients, as potential add-on therapy arose [6]. There has been a marked increase in the incidence of autoimmune diseases in the past half-a-century. Although the underlying genetic basis of this class of diseases has recently been elucidated, implicating predominantly immune-response genes, changes in environmental 
factors must ultimately be driving this increase [7].

\section{High Sodium Intake and MS Attacks}

Recently, salt has been shown to modulate the differentiation of human and mouse Th17 cells. Mice fed on a high-sodium diet were described to develop more aggressive courses of experimental autoimmune encephalomyelitis. However, the role of sodium intake in MS has not been addressed. For that reason, Farez et al. aimed to investigate the relationship between salt consumption and radiological and clinical disease activity in MS patients [6].

The study involved two separate groups of patients with MS. One group included 70 individuals with frequently relapsing MS was followed longitudinally for 2 years. The patients' sodium intake was measured from urine and serum samples and regression analysis was used to determine the effect of the intake on their disease activity [2]. The findings were then replicated in a separate group of 52 patients with MS.

\section{SGK1 a Major Player in the Induction of Pathogenic Th17 Cells}

Th17 cells IL-17 (interleukin-17)-producing helper $\mathrm{T}$ cells are highly proinflammatory cells, critical for clearing extracellular pathogens and for inducing multiple autoimmune diseases. IL-23 has a critical role in stabilizing and reinforcing the Th17 phenotype by increasing expression of IL-23R (IL-23 receptor) and endowing Th17 cells with pathogenic effector functions. However, the precise molecular mechanism by which IL-23 sustains the Th17 response and induces those pathogenic functions has not been elucidated. Transcriptional profiling was used to construct a model of developing Th17 cells of their signaling network and nominate major nodes that regulate Th17 development. The authors identified SGK1 (serum glucocorticoid kinase 1), a serine/threonine kinase, as an essential node downstream of IL-23 signaling. SGK1 is critical for regulating IL-23R expression and stabilizing the Th17 cell phenotype by deactivation of mouse Foxo1, a direct repressor of IL-23R expression. SGK1 has been shown to govern $\mathrm{Na}^{+}$transport and salt $(\mathrm{NaCl})$ homeostasis in other cells. It was shown that a modest increase in salt concentration could induce SGK1 expression, promote IL-23R expression and enhance Th17 cell differentiation in vitro and in vivo, accelerating the development of autoimmunity. Loss of SGK1 abrogated $\mathrm{Na}^{+}$-mediated Th17 differentiation in an IL-23-dependent manner.

These data demonstrate that SGK1 has a critical role in the induction of pathogenic Th17 cells and provide a molecular insight into a mechanism by which an environmental factor such as a high salt diet triggers Th17 development and promotes tissue inflammation [8].

The newly identified population of interleukin (IL)-17-producing CD4 (+) helper T cells (Th17 cells) has a pivotal role in autoimmune diseases. Pathogenic IL-23-dependent Th17 cells have been shown to be critical for the development of EAE (experimental autoimmune encephalomyelitis) in an animal model for multiple sclerosis, and genetic risk factors associated with multiple sclerosis are related to the IL-23-Th17 pathway. However, little is known about the environmental factors that directly influence Th17 cells. Increased salt (sodium chloride, $\mathrm{NaCl}$ ) concentrations found locally under physiological conditions in vivo markedly boost the induction of murine and human Th17 cells. High-salt conditions activate the p38/MAPK pathway involving nuclear factor of activated T cells 5 (NFAT5; also called TONEBP) and serum/glucocorticoid-regulated kinase 1 during cytokine-induced Th17 polarization. Gene silencing or chemical inhibition of p38/MAPK, NFAT5 or SGK1 abrogates the high-salt-induced Th17 cell development [7].

\section{Correlation between Exacerbation Rates and Sodium Intake}

High-salt diet induces Th17 cells in vivo and 
exacerbates autoimmune encephalomyelitis. Naive murine CD4 cells were stimulated with radiated APC, anti-CD3, IL-6 and TGF- $\beta 1$ in the presence $(\mathrm{NaCl})$ or absence of $\mathrm{NaCl}$ and were analysed by FACS. IL-17A secretion of primary splenocytes was measured by ELISA, after their stimulation by anti-CD3 in the presence or absence of $\mathrm{NaCl}$. Spinal cord from EAE animals was analysed by qRT-PCR. Splenocytes from EAE animals were analysed by qRT-PCR after they were re-stimulated with MOG for 2 days and supernatants were analysed for IL-17A and IFN- $\gamma$ by ELISA or cells were analysed for IL-17A by FACS. The authors found a positive correlation between exacerbation rates and sodium intake in a multivariate model adjusted for gender, age, disease duration, vitamin D levels, smoking status or body mass index. They discovered an exacerbation rate that was about three times more common in patients with medium salt intake or nearly four times higher for the group with high sodium intake compared to the low-intake group. Additionally, individuals with high-sodium intake had an increased chance of developing a new lesion on the MRI and on average had eight more T2 lesions on MRI. A similar relationship was found in the independent replication group [6].

The Th17 cells generated under high-salt conditions displayed a highly pathogenic and stable phenotype characterized by the upregulation of the pro-inflammatory cytokines GM-CSF, TNF- $\alpha$ and IL-2.

Moreover, mice fed with a high-salt diet developed a more severe form of EAE, in line with augmented central nervous system infiltrating and peripherally induced antigen-specific Th17 cells. Thus, increased dietary salt intake might represent an environmental risk factor for the development of autoimmune diseases through the induction of pathogenic Th17 cells [7].

\section{Conclusion}

These results suggest that higher sodium intake is associated with increased clinical manifestation of the disease activity in patients with MS. Larger studies are needed in order to adjust the salt consumption from MS patients or to prove the health benefits from reducing it.

\section{Acknowledgments}

This work was supported by the European Social Fund and Republic of Bulgaria, Operational Programme "Human Resources Development" 2007-2013 framework, Grant № BG051PO001-3.3.06-0048 from Oct.04.2012.

\section{References}

[1] Compston, A., and Coles, A. 2008. "Multiple sclerosis." Lancet 372 (9648): 1502-17.

[2] Melville, N. A. 2014. "High Sodium Intake Linked to Increased MS Exacerbations." Accessed September 3, 2014. http://www.medscape.com/viewarticle/830959.

[3] Riccio, P., and Rossano, R. 2015. "Nutrition facts in multiple sclerosis." ASN Neuro 7 (1).

[4] Johnson, S. 2001. "The multifaceted and widespread pathology of magnesium deficiency." Med. Hypotheses 56 (2): 163-70.

[5] Rayssiguier, Y., Gueux, E., Nowacki, W., Rock, E., and Mazur, A. 2006. "High fructose consumption combined with low dietary magnesium intake may increase the incidence of the metabolic syndrome by inducing inflammation.” Magnes. Res. 19 (4): 237-43.

[6] Farez, M. F., Fiol, M. P., Gaitán, M. I., Quintana, F. J., and Correale, J. 2015. "Sodium Intake Is Associated with Increased Disease Activity in Multiple Sclerosis." J. Neurol. Neurosurg. Psychiatry 86: 26-31. doi:10.1136/jnnp-2014-307928.

[7] Kleinewietfeld, M., Manzel, A., Titze, J., Kvakan, H., Yosef, N., Linker, R. A., Muller, D. N., and Hafler, D. A. 2013. "Sodium Chloride Drives Autoimmune Disease by the Induction of Pathogenic Th17 Cells." Nature 496 (7446): 518-22.

[8] Wu, C., Yosef, N., Thalhamer, T., Zhu, C., Xiao, S., Kishi, Y., Regev, A., and Kuchroo, V. K. 2013. "Induction of Pathogenic Th17 Cells by Inducible Salt-Sensing Kinase SGK1.” Nature 496 (7446): 513-7. 